\title{
OPTIMAL STOCHASTIC SWTTCHING AND THE DIRICHLET PROBLEM FOR THE BELLMAN EQUATION
}

BY

\author{
LAWRENCE C. EVANS AND AVNER FRIEDMAN ${ }^{1}$
}

\begin{abstract}
Let $L^{i}$ be a sequence of second order elliptic operators in a bounded $n$-dimensional domain $\Omega$, and let $f^{i}$ be given functions. Consider the problem of finding a solution $u$ to the Bellman equation $\sup _{i}\left(L_{i} u-f^{i}\right)$ $=0$ a.e. in $\Omega$, subject to the Dirichlet boundary condition $u=0$ on $\partial \Omega$. It is proved that, provided the leading coefficients of the $L^{i}$ are constants, there exists a unique solution $u$ of this problem, belonging to $W^{1, \infty}(\Omega) \cap$ $W_{\text {loc }}^{2, \infty}(\Omega)$. The solution is obtained as a limit of solutions of certain weakly coupled systems of nonlinear elliptic equations; each component of the vector solution converges to $u$. Although the proof is entirely analytic, it is partially motivated by models of stochastic control. We solve also certain systems of variational inequalities corresponding to switching with cost.
\end{abstract}

1. Introduction. Consider a sequence of linear elliptic partial differential operators

$$
L^{k} u \equiv-\sum_{i j=1}^{n} a_{i j}^{k}(x) \frac{\partial^{2} u}{\partial x_{i} \partial x_{j}}-\sum_{i=1}^{n} b_{i}^{k}(x) \frac{\partial u}{\partial x_{i}}+c^{k}(x) u \quad(k=1,2, \ldots)
$$

in a bounded domain $\Omega \subset R^{n}$. We assume:

$$
\begin{gathered}
\partial \Omega \in C^{2+\beta} \quad \text { for some } \beta>0, \\
\sum_{i, j=1}^{n} a_{i j}^{k}(x) \xi_{i} \xi_{j}>\gamma|\xi|^{2} \quad\left(x \in \Omega, \xi \in R^{n}, \gamma>0\right), \\
\left|D^{\alpha} a_{i j}^{k}(x)\right|,\left|D^{\alpha} b_{i}^{k}(x)\right|,\left|D^{\alpha} c^{k}(x)\right|<C \\
(x \in \Omega, 1 \leqslant i, j \leqslant n ; 0 \leqslant|\alpha|<2, C>0),
\end{gathered}
$$

and

$$
c^{k}(x) \geqslant c_{0} \quad\left(x \in \Omega ; c_{0}>0\right),
$$

where $\gamma, C, c_{0}$ are constants independent of $k$. (The hypothesis $c_{0}>0$ is convenient, but $c_{0} \geqslant 0$ is sufficient; see Remark 1, §6.)

Received by the editors April 16, 1978.

AMS (MOS) subject classifications (1970). Primary 35J60, 49A20; Secondary 35J25, 47H05, 47H15, 60H10, 93E20.

Key words and phrases. Bellman equation, elliptic operators, nonlinear elliptic equation, accretive operators, stochastic differential equations.

${ }^{1}$ This work is partially supported by National Science Foundation Grants MCS 77-09152 and MC 575-21416 A01.

C 1979 American Mathematical Society $0002-9947 / 79 / 0000-0417 / \$ 07.25$ 
Suppose further that the $f^{k}(x)$ are given functions for $x \in \Omega$, satisfying

$$
\left|D^{\alpha} f^{k}(x)\right| \leqslant C \quad(x \in \Omega ; 0 \leqslant|\alpha| \leqslant 2, C>0),
$$

where $C$ is a constant independent of $k$.

In this paper we investigate the nonlinear partial differential equation

$$
\sup _{k}\left(L^{k} u(x)-f^{k}(x)\right)=0 \quad \text { a.e. for } x \in \Omega,
$$

with the boundary condition

$$
u=0 \text { on } \partial \Omega \text {. }
$$

Equation (1.6) is called the Bellman equation of dynamic programming, arising in stochastic optimal control theory (see \$7). It was studied by Krylov [13] and Nisio [14] in the case $\Omega=R^{n}$. Krylov proved (under assumptions similar to (1.2)-(1.5) and with the additional condition that $c_{0}$ is sufficiently large) that there exists a unique solution $u$ in $R^{n}$ such that, for some $\varphi(x)=\left(1+\mu|x|^{2}\right)^{1 / 2}, \mu>0, e^{-\varphi(x)} u(x)$ belongs to $W^{2, p}\left(R^{n}\right), \forall p<\infty$. His proof was somewhat simplified by Nisio [14] (see also [3]). As is pointed out in [14], [3], the existence of a solution to the Bellman equation in a bounded domain is an open problem, except when $n=2$.

In this paper we establish the existence of a unique solution $u$ of (1.6), (1.7) satisfying $u \in W^{1, \infty}(\Omega) \cap W_{\mathrm{loc}}^{2, \infty}(\Omega)$. We assume the conditions (1.1)-(1.5) and the additional restriction

$$
a_{i j}^{k} \text { are constants. }
$$

Unlike Krylov's approach, our proof does not use probabilistic methods; it is however partially motivated by probabilistic considerations. In fact, we approximate the solution of (1.6), (1.7) by the system of equations

$$
\begin{aligned}
L^{k} u^{k}+\beta_{e}\left(u^{k}-u^{k+1}\right) & =f^{k}(x) \quad(1 \leqslant k \leqslant m, \quad x \in \Omega), \\
u^{k} & =0 \text { on } \partial \Omega,
\end{aligned}
$$

where $u^{m+1} \equiv u^{1}$. Here $\beta_{\varepsilon}$ represents a "penalty" term: $\beta_{\varepsilon}(t)=0$ if $t<0$, $\beta_{\varepsilon}(t) \rightarrow \infty$ if $t>0, \varepsilon \rightarrow 0$. Probabilistically, the solution component $u^{k}(x)$ represents the optimal cost starting at $x \in \Omega$ in state $k$ of the same cost functional as in Krylov's work plus an additional cost for every switching from one generator $L^{i}$ to the next one $L^{i+1}$. We shall explain this model more precisely in $\$ 7$.

In $\$ 2$ we study the system (1.9) and prove that it has a unique classical solution. $\$ 3$ comprises an a priori estimate on the $W^{1, \infty}(\Omega)$-norm of the solution; the estimate does not depend on $m$ and $\varepsilon$. In $\$ 4$ we derive a priori estimate on the $W^{2, \infty}\left(\Omega_{0}\right)$-norm of the solution of (1.9), $\bar{\Omega}_{0} \subset \Omega$. It is only here that the condition (1.8) is used.

In $\S 5$ we take $\varepsilon \rightarrow 0$ in (1.9) and show that each $u^{k}=u^{k, e}$ converges to the same function $v^{m}, v^{m}$ solving the Bellman problem (1.6), (1.7) (where $k$ 
ranges over the indices $1,2, \ldots, m)$. Finally, in $\$ 6$ we prove that, as $m \rightarrow \infty$, $v^{m}$ converges to the solution of (1.6), (1.7). These convergence arguments use nonlinear functional analytic methods and, in particular, certain accretive operator techniques.

In $\$ 7$ we study the system

$$
\begin{gathered}
L^{i} u_{i}+\beta_{e}\left(u_{i}-u_{i+1}-k_{i}\right)=f_{i} \quad(x \in \Omega), \\
u_{i}=0 \quad \text { on } \partial \Omega \quad(1 \leqslant i \leqslant m)
\end{gathered}
$$

where the $k_{i}$ are positive numbers, and also the limit case, as $\varepsilon \rightarrow 0$, a system of variational inequalities

$$
\begin{gathered}
L^{i} u_{i}+\beta\left(u_{i}-u_{i+1}-k_{i}\right) \ni f^{i} \text { a.e. in } \Omega, \\
u_{i}=0 \text { on } \partial \Omega .
\end{gathered}
$$

(Here $\beta(t)$ is the maximal monotone graph: $\beta(t)=\{0\}$ if $t<0, \beta(0)=$ $[0, \infty]$.) We give probabilistic interpretation for these problems and prove that each component $u_{i}$ of the solution of (1.11) converges to the solution $v^{m}$ (of (1.6), (1.7) for $\left.L^{1}, \ldots, L^{m}\right)$ as $\left(k_{1}, \ldots, k_{m}\right) \rightarrow 0$.

We also show that for fixed and positive $k_{i}$, the solution of (1.10) converges to the solution of (1.11), as $\varepsilon \rightarrow 0$. This is proved under weaker assumptions than (1.3), (1.5) and without the restrictive condition (1.8).

In $\S 8$ we specialize to the case $m=2$. It was proved by Brezis and Evans [5] that the corresponding Bellman equation

$$
\begin{gathered}
\max \left(L^{1} u(x)-f^{1}(x), L^{2} u(x)-f^{2}(x)\right)=0 \text { a.e. in } \Omega, \\
u=0 \text { on } \partial \Omega
\end{gathered}
$$

has a solution $u$ in $C^{2+\alpha}\left(\Omega_{0}\right)$ for some $\alpha>0$ and any domain $\Omega_{0}, \bar{\Omega}_{0} \subset \Omega$. Here we show (without making the restriction (1.8)) that the solution $u_{1}, u_{2}$ of (1.11) with $m=2$ satisfies: $u_{i}(x) \rightarrow u(x)$ as $\left(k_{1}, k_{2}\right) \rightarrow 0$.

NOTE ADDED IN PROOF. P. L. Lions (in work to appear) has recently removed the restriction hypothesis (1.8). His method gives $W^{2, \infty}(\Omega)$ estimates for the approximating system (2.3), (2.4) and is based on a nontrivial extension of our proof of Lemma 4.1.

2. Construction of approximate solutions. Let $\beta_{e}(t)\left(\varepsilon>0, t\right.$ real) be a $C^{\infty}$ function in $t$ such that

$$
\begin{aligned}
& \beta_{e}(t)=0 \quad \text { if } t \leqslant 0, \\
& \beta_{e}(t) \rightarrow \infty \quad \text { if } t>0, \quad \varepsilon \rightarrow 0, \\
& \beta_{e}^{\prime}(t) \geqslant 0, \quad \beta_{e}^{\prime \prime}(t) \geqslant 0,
\end{aligned}
$$

and

$$
\left|t \beta_{e}^{\prime}(t)-\beta_{\varepsilon}(t)\right| \leqslant C \quad(C \text { constant }) .
$$


We can take, for example,

and

$$
\begin{aligned}
\beta_{\varepsilon}(t) & =\frac{t-\varepsilon}{\varepsilon} \quad \text { if } 2 \varepsilon<t<\infty, \\
& =0 \text { if } t \leqslant 0,
\end{aligned}
$$

$$
0<\beta_{\varepsilon}(t)<1, \quad 0 \leqslant \beta_{\varepsilon}^{\prime}(t) \leqslant \frac{1}{\varepsilon} \quad \text { if } 0<t<2 \varepsilon .
$$

Consider now the semilinear elliptic system

$$
\begin{gathered}
L^{k} u^{k}+\beta_{e}\left(u^{k}-u^{k+1}\right)=f^{k} \quad \text { in } \Omega \quad(1<k<m), \\
u^{k}=0 \text { on } \partial \Omega
\end{gathered}
$$

where $u^{m+1} \equiv u^{1}$.

This system can be solved by several standard methods. It is most convenient for our purposes to invoke certain facts of nonlinear functional analysis, as these considerations are crucial for the convergence results of $\$ 5$. We begin by recalling some definitions; for more details, see [1].

In any real Banach space $X$ one defines the pairing

$$
[x, y]_{+} \equiv \inf _{\lambda>0} \frac{\|x+\lambda y\|-\|x\|}{\lambda} \quad(x, y \in X) \text {. }
$$

It can be shown that

$$
\lim _{\lambda \downarrow 0} \frac{\|x+\lambda y\|-\|x\|}{\lambda} \text { exists and equals }[x, y]_{+} .
$$

Note that

$$
[,]_{+}: X \times X \rightarrow R \text { is upper semicontinuous. }
$$

A nonlinear operator $A$ with domain $D(A)$ in $X$ and range in $X$ is called accretive if

$$
\|x-y\| \leqslant\|x-y+\lambda(A x-A y)\| \quad \forall x, y \text { in } D(A), \quad \lambda>0,
$$

and $m$-accretive if, in addition, $R(I+\lambda A)=X$ for all $\lambda>0$ (or, equivalently, for some $\lambda>0$ ). $A$ is accretive if and only if

$$
[x-y, A x-A y]_{+}>0 \quad \forall x, y \text { in } D(A) .
$$

If $X=C(\bar{\Omega})$, the brackets [ , ] admit the representation

$$
[f, g]_{+}=\max _{\substack{y \in \Omega \\|f(y)|=\|f\|}} g(y) \cdot \operatorname{sgn} f(y) \quad(f \neq 0),
$$

see, for example, Sato [16, p. 431] or Sinestrari [17, p. 22]. As we shall see, this characterization is useful for studying partial differential equations satisfying a maximum principle.

The following lemma is due to Frank Massey (unpublished).

LEMMA 2.1. Let $X=X^{1} \times \cdots \times X^{n}$ be the product of real Banach spaces 
$X^{i}$ with norm

$$
\|x\|=\max _{1<i<n}\left\|x^{i}\right\| \quad\left(x=\left(x^{1}, \ldots, x^{n}\right)\right) .
$$

Denote by $[,]_{+}^{i}$ and $[,]_{+}$the brackets in $X^{i}$ and $X$, respectively. Then

$$
[x, y]_{+}=\max _{1<i<n}\left\{\left[x^{i}, y^{i}\right]_{+}^{i} ;\left\|x^{i}\right\|=\|x\|\right\} \text {. }
$$

Proof. If $\left\|x^{i}\right\|=\|x\|$, then

$$
\|x+\lambda y\|-\|x\|>\left\|x^{i}+\lambda y^{i}\right\|-\left\|x^{i}\right\| .
$$

Dividing by $\lambda$ and taking the infimum over $\lambda$, we get

$$
[x, y]_{+}>\left[x^{i}, y^{i}\right]_{+}^{i}
$$

This proves the inequality

$$
[x, y]_{+}>\max _{i}\left\{\left[x^{i}, y^{i}\right]_{+}^{i} ;\left\|x^{i}\right\|=\|x\|\right\}
$$

To prove the reverse, we take a sequence $\lambda_{m}>0$ such that $\lambda_{m} \downarrow 0$ and

$$
\left\|x+\lambda_{m} y\right\|=\left\|x^{i}+\lambda_{m} y^{i}\right\| \text { for some fixed } i \text {. }
$$

Then $\|x\|=\left\|x^{i}\right\|$ and

$$
\left\|x+\lambda_{m} y\right\|-\|x\|=\left\|x^{i}+\lambda_{m} y^{i}\right\|-\left\|x^{i}\right\| \text {. }
$$

Dividing by $\lambda_{m}$ and taking $\lambda_{m} \downarrow 0$, we obtain the opposite inequality to (2.8).

We now take $X^{i}=C(\bar{\Omega}), X=X^{1} \times \cdots \times X^{m}$ and introduce the vector notation

$$
u=\left(\begin{array}{c}
u^{1} \\
u^{2} \\
\vdots \\
u^{m}
\end{array}\right), \quad L u=\left(\begin{array}{c}
L^{1} u^{1} \\
L^{2} u^{2} \\
\vdots \\
L^{m} u^{m}
\end{array}\right), \quad B_{e} u=\left(\begin{array}{c}
\beta_{e}\left(u^{1}-u^{2}\right) \\
\beta_{e}\left(u^{2}-u^{3}\right) \\
\vdots \\
\beta_{e}\left(u^{m}-u^{1}\right)
\end{array}\right) .
$$

Each operator $L^{i}$ is defined on the set

$$
D\left(L^{i}\right)=\left\{v \in W_{0}^{1,2}(\Omega) \cap W^{2 p}(\Omega) ; L^{i} v \in C(\bar{\Omega})\right\}
$$

for any fixed $p>n$, and $L$ is defined on $D(L)=D\left(L^{1}\right) \times \cdots \times D\left(L^{m}\right)$. The operator $B_{\varepsilon}$ is defined everywhere on $X$.

LEMMA 2.2. The operator $A_{e}=L-c_{0} I+B_{e}$ with domain $D(L)$ is m-accretive in $X$.

Proof. According to a standard perturbation theory [1] it suffices to prove that

$$
B_{e} \text { is Lipschitz and accretive }
$$


and

$$
L-c_{0} I \text { is } m \text {-accretive. }
$$

Since $0 \leqslant \beta_{\varepsilon}^{\prime} \leqslant 1 / \varepsilon, B_{\varepsilon}$ is clearly Lipschitz. Furthermore, by Lemma 2.1,

$$
\begin{aligned}
& {\left[u-\bar{u}, B_{e} u-B_{e} \bar{u}\right]_{+}} \\
& \quad=\max \left\{\left[u^{i}-\bar{u}^{i}, \beta_{e}\left(u^{i}-u^{i+1}\right)-\beta_{e}\left(\bar{u}^{i}-\bar{u}^{i+1}\right)\right]_{+},\right. \\
& \left.\left\|u^{i}-\bar{u}^{i}\right\|=\|u-\bar{u}\|\right\} .
\end{aligned}
$$

Take $i$ such that $\left\|u^{i}-\bar{u}^{i}\right\|=\|u-\bar{u}\|$ and, without loss of generality, assume that

$$
\left\|u^{i}-\bar{u}^{i}\right\|=u^{i}\left(x^{0}\right)-\bar{u}^{i}\left(x^{0}\right) \text { for some } x^{0} \in \bar{\Omega} \text {. }
$$

Then

$$
u^{i}\left(x^{0}\right)-\bar{u}^{i}\left(x^{0}\right) \geq u^{i+1}\left(x^{0}\right)-\bar{u}^{i+1}\left(x^{0}\right)
$$

and so

$$
u^{i}\left(x^{0}\right)-u^{i+1}\left(x^{0}\right) \geqslant \bar{u}^{i}\left(x^{0}\right)-\bar{u}^{i+1}\left(x^{0}\right) .
$$

Because of the monotonicity of $\beta_{e}$, it follows that

$$
\beta_{e}\left(u^{i}-u^{i+1}\right) \geqslant \beta_{e}\left(\bar{u}^{i}-\bar{u}^{i+1}\right) \text { at } x^{0} .
$$

Recalling (2.7), we conclude that the right-hand side of (2.11) is nonnegative. This proves (2.9).

The proof that $L^{i}-c_{0} I$ is accretive follows by the maximum principle, making use of (2.7). In fact, if $v \in C(\bar{\Omega}) \cap W_{\mathrm{loc}}^{2 p}(\Omega)$ and $v$ takes a positive maximum at a point $x^{0} \in \Omega$, then by Bony [4] (see also [8])

$$
\text { ess } \lim _{x \rightarrow x^{0}} \inf \left(L^{i} v(x)-c_{0} v(x)\right)>0 .
$$

Since, in particular, $L^{i} v-c_{0} v$ is continuous, then $v\left(x^{g}\right)\left(L^{i} v\left(x^{g}\right)-c_{0} v\left(x^{g}\right)\right)>$ 0. Using this and (2.7), it is clear that $L^{i}-c_{0} I$ is accretive. Employing Lemma 2.1 we find that also $L-c_{0} I$ is accretive.

By the general theory of elliptic equations, each $L^{i}-c_{0} I$ is $m$-accretive; consequently $L-c_{0} I$ is also $m$-accretive.

THEOREM 2.3. Let (1.1)-(1.5) hold. Then there exists a unique solution of (2.3), (2.4) with components $u^{k}$ in $D\left(L^{k}\right)$; further, $u^{k}$ belongs to $C^{2, \beta}(\bar{\Omega}) \cap$ $C^{3, \theta}\left(\Omega_{0}\right)$ for any $0<\theta<1, \bar{\Omega}_{0} \subset \Omega$.

The first part follows from the $m$-accretiveness of $A_{\varepsilon}$. The second part is a consequence of standard regularity results for elliptic equations.

3. $W^{1, \infty}$ estimates. In this section we derive uniform estimates on the solution $u=\left(u^{1}, \ldots, u^{m}\right)$ of (2.3), (2.4) and on its first derivatives; these bounds will not depend on the parameters $\varepsilon, m$. The condition (1.8) will not be needed here. 
LEMMA 3.1.

$$
\max _{k}\left\|u^{k}\right\|_{L^{\infty}(\Omega)} \leqslant \frac{1}{c_{0}} \max _{k}\left\|f^{k}\right\|_{L^{\infty}(\Omega)}
$$

Proof. We first prove that

$$
u^{k}(x) \geqslant-\frac{1}{c_{0}} \max _{k}\left\|f^{k}\right\|_{L^{\infty}(\Omega)} \quad(x \in \Omega) .
$$

Denote by $N_{k}$ the minimum of $u^{k}$ in $\bar{\Omega}$ and choose $j$ such that $N_{j}=\min N_{k}$. Without loss of generality we may suppose that $N_{j}<0$. Let $x^{0}$ be a point in $\Omega$ such that $u^{j}\left(x^{g}\right)=N_{j}$. Then

$$
\begin{gathered}
L^{j} u^{j}\left(x^{0}\right) \leqslant c^{j}\left(x^{0}\right) u^{j}\left(x^{0}\right)=c^{j}\left(x^{0}\right) N_{j} \leqslant c_{0} N_{j}, \\
\beta_{e}\left(u^{j}\left(x^{0}\right)-u^{j+1}\left(x^{0}\right)\right)=0
\end{gathered}
$$

(since $u^{j}\left(x^{9}\right)<u^{j+1}\left(x^{9}\right)$ ). Consequently,

$$
c_{0} N_{j} \geqslant L^{j} u^{j}\left(x^{0}\right)+\beta_{\varepsilon}\left(u^{j}\left(x^{0}\right)-u^{j+1}\left(x^{0}\right)\right)=f^{j}\left(x^{0}\right),
$$

so that

$$
N_{j} \geqslant-\frac{1}{c_{0}}\left\|f_{j}\right\|_{L^{\infty}(\Omega)}
$$

this proves (3.2). Similarly one estimates the $u^{k}$ from above.

In what follows we shall denote by $C$ a generic positive constant which is independent of $m, \varepsilon$.

LEMMA 3.2.

$$
\left\|D u^{k}\right\|_{L^{\infty}(\partial \Omega)} \leqslant C \quad(1 \leqslant k \leqslant m)
$$

Proof. Let $\bar{v}^{k}$ be the solution of

$$
\begin{gathered}
L^{k} \bar{v}^{k}=f^{k} \quad \text { in } \Omega, \\
\bar{v}^{k}=0 \text { on } \partial \Omega .
\end{gathered}
$$

Since $L^{k} u^{k} \leqslant f^{k}$, the maximum principle implies that $u^{k}<\bar{v}^{k}$. It follows that

$$
\frac{\partial u^{k}}{\partial \nu} \leqslant \frac{\partial \bar{v}^{k}}{\partial \nu} \leqslant C \quad \text { on } \partial \Omega
$$

where $\nu$ is the inner normal.

Next, let $x^{0}$ be a point on $\partial \Omega$ and let $y^{0}$ be the center of a ball $B$ of radius $R$ such that $\bar{B} \cap \bar{\Omega}=\left\{x^{0}\right\} ; R$ can be taken to be independent of $x^{0}$. For sufficiently large positive constants $p$ and $D$, the function

$$
w(x)=D\left(\frac{1}{R^{p}}-\frac{1}{r^{p}}\right) \quad\left(r=\left|x-y^{0}\right|\right)
$$

is a barrier at $x^{0}$ for each $L^{i}$; that is, $w=0$ at $x^{0}, w>0$ in $\bar{\Omega} \backslash\left\{x^{0}\right\}$, and $L^{i} w>1$ (the constants $p, D$ are independent of $i$ ). 
Let $v^{k} \equiv-C w$ for $1<k \leqslant m$, for $C>\max _{L}\left\|f^{k}\right\|_{L^{\infty}(\Omega)}$. Then

$$
\begin{gathered}
L^{k} v^{k}+\beta_{e}\left(v^{k}-v^{k+1}\right)=g^{k} \text { in } \Omega, \\
v^{k} \leqslant 0 \text { on } \partial \Omega,
\end{gathered}
$$

and $g^{k}=-C L^{k} w<f^{k}$. We claim that

$$
v^{k}<u^{k} \text { for all } k, x \in \Omega \text {. }
$$

Indeed, otherwise choose $j$ such that

$$
\max _{\Omega}\left(v^{k}(x)-u^{k}(x)\right) \equiv M_{k}
$$

is the largest when $k=j$, and let $x^{*} \in \Omega$ be such that

$$
\begin{gathered}
0<M_{j}=v^{j}\left(x^{*}\right)-u^{j}\left(x^{*}\right) . \\
\text { At } x=x^{*}, L^{j}\left(v^{j}-u^{j}\right) \geqslant c^{j}\left(v^{j}-u^{j}\right)>0, g^{j}-f^{j}<0, \text { and consequently } \\
\beta_{e}\left(v^{j}-v^{j+1}\right)<\beta_{e}\left(u^{j}-u^{j+1}\right) .
\end{gathered}
$$

Noting that $v^{j} \equiv v^{j+1}$, we deduce that $u^{j}\left(x^{*}\right)-u^{j+1}\left(x^{*}\right)>0$. Hence

$$
\begin{aligned}
\max _{\Omega}\left(v^{j+1}-u^{j+1}\right) & >v^{j+1}\left(x^{*}\right)-u^{j+1}\left(x^{*}\right)>v^{j}\left(x^{*}\right)-u^{j}\left(x^{*}\right) \\
& =\max _{\Omega}\left(v^{j}-u^{j}\right),
\end{aligned}
$$

thus contradicting the definition of $j$.

Having proved (3.6), we now notice that $v^{k}=u^{k}=0$ at $x^{0}$. Hence

$$
\frac{\partial u^{k}}{\partial \nu}>\frac{\partial v^{k}}{\partial \nu}=-C \frac{\partial w}{\partial \nu} \text { at } x^{0}
$$

This, together with (3.4), complete the proof of (3.3).

LEMMA 3.3.

$$
\left\|D u^{k}\right\|_{L^{\infty}(\Omega)}<C \quad(1<k<m) .
$$

Proof. Let $\lambda$ be a positive number to be determined later on (independently of $m, \varepsilon$ ), and choose $j$ such that

$$
\max _{\Omega}\left[\left|\nabla u^{j}\right|^{2}+\lambda\left(u^{j}\right)^{2}\right]>\max _{\Omega}\left[\left|\nabla u^{k}\right|^{2}+\lambda\left(u^{k}\right)^{2}\right]
$$

for all $k$. We take $j=1$ for simplicity and write $u=u^{1}, L=L^{1}, a_{i j}=a_{i j}^{1}$, $b_{i}=c_{i}^{1}, c=c^{1}, f=f^{1}$ and $\beta=\beta_{e}$. We also set $v=u^{2}$.

We shall denote partial derivatives by subscripts and use the summation convention.

We shall estimate the function

$$
w=|\nabla u|^{2}+\lambda u^{2}=u_{i} u_{i}+\lambda u^{2}
$$

by applying to it the maximum principle. First we compute

$$
\begin{gathered}
w_{\mu}=2 u_{i} u_{i \mu}+2 \lambda u u_{\mu}, \\
w_{\mu \nu}=2 u_{i \nu} u_{i \mu}+2 u_{i} u_{i \mu \nu}+2 \lambda u_{\nu} u_{\mu}+2 \lambda u u_{\mu \nu} .
\end{gathered}
$$


Differentiating the equation

$$
L u+\beta(u-v)=f
$$

we obtain

$$
L u_{i}+\beta^{\prime}(u-v)\left(u_{i}-v_{i}\right)=f_{i}+\tilde{D}^{2} u \quad(1<i \leqslant n)
$$

where

$$
\tilde{D}^{2} u=\sum_{|\alpha|<2} \sigma_{\alpha} D^{\alpha} u, \quad \sigma_{\alpha} \text { bounded. }
$$

We now compute $L w$, by substituting from (3.9)

$$
\begin{aligned}
L w= & -2 a_{\mu \nu} u_{i \mu} u_{i \nu}-2 u_{i} a_{\mu \nu} u_{i \mu \nu}-2 \lambda a_{\mu \nu} u_{\mu} u_{\nu} \\
& -2 \lambda u a_{\mu \nu} a_{\mu \nu}-2 u_{i} b_{\mu} u_{i \mu}-2 \lambda u b_{\mu} u_{\mu}+c u_{i} u_{i}+\lambda c u^{2} \\
\leqslant & -2 \gamma u_{i \mu} u_{i \mu}-2 \gamma \lambda u_{\mu} u_{\mu}+2 u_{i} L u_{i}+2 \lambda u L u .
\end{aligned}
$$

Recalling (3.10), (3.11), we get

$$
\begin{aligned}
L w \leqslant & -2 \gamma u_{i j} u_{i j}-2 \gamma \lambda u_{i} u_{i}+2 u_{i} f_{i}+2 u_{i} \tilde{D}^{2} u+2 \lambda u f \\
& -2 u_{i} \beta^{\prime}(u-v)\left(u_{i}-v_{i}\right)-2 \lambda u \beta(u-v) .
\end{aligned}
$$

Since

$$
\left|2 u_{i} \tilde{D}^{2} u\right| \leqslant \gamma u_{i j} u_{i j}+C u_{i} u_{i}
$$

and since $|u| \leqslant C$, we obtain, upon choosing $\lambda$ to be sufficiently large (depending only on $C$ )

$$
L w \leqslant C-J
$$

where

$$
J=2 \lambda u \beta(u-v)+2 \beta^{\prime}(u-v) u_{i}\left(u_{i}-v_{i}\right) .
$$

Suppose the maximum of $w$ in $\bar{\Omega}$ is attained at a point $x^{0} \in \Omega$. Then

$$
u_{i} u_{i}+\lambda u^{2}>v_{i} v_{i}+\lambda v^{2} \text { at } x=x^{0}
$$

(since (3.8) holds with $j=1$ ). Next, because of (2.2),

$$
J>2 \beta^{\prime}(u-v) u_{i}\left(u_{i}-v_{i}\right)+2 \lambda u \beta^{\prime}(u-v)(u-v)-C .
$$

Noting that

$$
\begin{aligned}
2 u_{i}\left(u_{i}-v_{i}\right) & \geqslant u_{i} u_{i}-v_{i} v_{i} \\
2 u(u-v) & \geqslant u^{2}-v^{2}
\end{aligned}
$$

we obtain

$$
J>\beta^{\prime}(u-v)\left(u_{i} u_{i}-v_{i} v_{i}+\lambda u^{2}-\lambda u^{2}\right)-C .
$$

Hence, by (3.14), $J>-C$ at $x=x^{0}$. Thus, by (3.12), $L w\left(x^{9}\right)<C$. But since $w$ attains it maximum in $\bar{\Omega}$ at $x^{0}$, we must have

$$
\operatorname{Lw}\left(x^{0}\right)>c\left(x^{0}\right) w\left(x^{0}\right)>c_{0} w\left(x^{0}\right) .
$$

Therefore $w\left(x^{0}\right)<C / c_{0}$. 
If $w$ attains its maximum only at boundary points, then the assertion $\max _{\bar{\Omega}} w \leqslant C$ follows from Lemma 3.2. This completes the proof of (3.7).

4. $W^{2, \infty}$ estimates. In this section we require also, in addition to (1.1)-(1.5), the condition (1.8). As before, $C$ will denote a generic constant independent of $\varepsilon, m$.

LEMMA 4.1. For any compact subdomain $\Omega_{0}$ of $\Omega$

$$
\left\|u^{k}\right\|_{W^{2, \infty}\left(\Omega_{0}\right)} \leqslant C \quad(1 \leqslant k \leqslant m)
$$

where $C$ depends on $\Omega_{0}$.

Proof. Without loss of generality we may assume that the $u^{k}$ belong to $C^{4}(\Omega)$. Indeed, otherwise we approximate $L^{i}$ by $L^{i, q}$ with $C^{3}$ coefficients (say); the derivation of (4.1) for the corresponding solution $u^{k, q}$ (given below) shows that $C$ depends only on the constants occurring in (1.2)-(1.5) and thus can be taken to be independent of $q$. Taking $q \rightarrow \infty$, the assertion (4.1) then follows.'

Let $\xi$ denote any direction and let $\zeta$ be a function in $C_{0}^{\infty}(\Omega)$ such that $\zeta=1$ in $\Omega_{0}, \zeta \geqslant 0$ elsewhere. Consider the numbers

$$
M_{i}=\max _{\Omega}\left\{\zeta^{2}\left(\left(u_{\xi \xi}^{i}\right)^{+}\right)^{2}+\lambda\left|\nabla u_{i}\right|^{2}\right\} \quad(\lambda>0)
$$

and suppose for definiteness that

$$
M_{1} \geqslant M_{i} \text { for all } 1 \leqslant i<k \text {; }
$$

here $\lambda$ is a positive number (independent of $\varepsilon, m$ ) to be determined later on. As in the proof of Lemma 3.3, we set $u=u^{1}, v=u^{2}, L=L^{1}, f=f^{1}$ and $\beta=\beta_{\varepsilon}$.

Differentiating (3.10) twice with respect to $\xi$, we get, using (1.8),

$$
L u_{\xi \xi}+\beta^{\prime}(u-v)\left(u_{\xi \xi}-v_{\xi \xi}\right)+\beta^{\prime \prime}(u-v)\left(u_{\xi}-v_{\xi}\right)^{2}=f_{\xi \xi}+\tilde{D}^{2} u
$$

where

$$
\tilde{D}^{2} u=\sum_{|\alpha|<2} \sigma_{\alpha} D^{\alpha} u, \quad \sigma_{\alpha} \text { bounded. }
$$

Since $\beta^{\prime \prime} \geqslant 0$, we conclude that

$$
L u_{\xi \xi}+\beta^{\prime}(u-v)\left(u_{\xi \xi}-v_{\xi \xi}\right) \leqslant f_{\xi \xi}+\tilde{D}^{2} u .
$$

Consider now the function

$$
w=\zeta^{2}\left(\left(u_{\xi \xi}\right)^{+}\right)^{2}+\lambda|\nabla u|^{2}
$$

and suppose that it attains its maximum in $\bar{\Omega}$ at a point $x^{0}$. If $x^{0} \in \partial \Omega$ then

$$
w\left(x^{0}\right)=\lambda\left|\nabla u\left(x^{0}\right)\right|^{2} \leqslant C,
$$

by Lemma 3.2. Suppose next that $x^{0} \in \Omega$. If $u_{\xi \xi}\left(x^{9}\right) \leqslant 0$, then again (4.5) holds, by Lemma 3.3 . 
We shall now consider the case where $u_{\xi \xi}\left(x^{0}\right)>0$. Then $u_{\xi \xi}>0$ in a neighborhood $G$ of $x^{0}$, and thus

$$
w=\zeta^{2}\left(u_{\xi \xi}\right)^{2}+\lambda|\nabla u|^{2}, \quad u_{\xi \xi}>0 \text { in } G .
$$

We now compute

$$
\begin{aligned}
w_{i}= & 2 u_{\xi \xi} u_{\xi \xi i} \zeta^{2}+2 \zeta \zeta_{i} u_{\xi \xi}^{2}+2 \lambda u_{k} u_{k i}, \\
w_{i j}= & 2 u_{\xi \xi i} u_{\xi \xi j} \zeta^{2}+2 u_{\xi \xi} u_{\xi \xi i j} \zeta^{2}+4\left(u_{\xi \xi} u_{\xi \xi i} \zeta \zeta_{j}+u_{\xi \xi} u_{\xi \xi j} \zeta \zeta_{i}\right) \\
& +2 u_{\xi \xi}^{2}\left(\zeta \zeta_{i j}+\zeta_{i} \zeta_{j}\right)+2 \lambda u_{k j} u_{k i}+2 \lambda u_{k} u_{k i j} .
\end{aligned}
$$

It follows easily that

$$
\begin{aligned}
-a_{i j} w_{i j} \leqslant & -2 \zeta^{2} a_{i j} u_{\xi \xi i} u_{\xi \xi j}-2 \zeta^{2} u_{\xi \xi} a_{i j} u_{\xi \xi i j} \\
& +C \zeta \sum_{i}\left|u_{\xi \xi i} u_{\xi \xi}\right|+C u_{\xi \xi}^{2}-2 \lambda a_{i j} u_{k i} u_{k j}-2 \lambda u_{k} a_{i j} u_{k i j}, \\
& -b_{i} w_{i} \leqslant 2 \zeta^{2} u_{\xi \xi} b_{i} u_{\xi \xi i}+C \zeta u_{\xi \xi}^{2}+2 \lambda u_{k} b_{i} u_{k i} .
\end{aligned}
$$

Hence

$$
\begin{aligned}
L w \leqslant & -2 \gamma \zeta^{2} u_{\xi \xi i} u_{\xi \xi i}+2 \zeta^{2} u_{\xi \xi} L u_{\xi \xi} \\
& +\left(\gamma \zeta^{2} u_{\xi \xi i} u_{\xi \xi i}+\frac{C}{\gamma} u_{\xi \xi}^{2}\right)-2 \lambda \gamma u_{k i} u_{k j}+2 \lambda u_{k} L u_{k} .
\end{aligned}
$$

We now specialize to $x=x^{0}$. Since $u_{\xi \xi}\left(x^{9}\right)>0$, we can substitute $L u_{\xi \xi}$ from (4.4) and still preserve the last inequality. Substituting afso $L u_{k}$ from (3.11), we get

$$
\begin{aligned}
L w \leqslant & -\gamma \zeta^{2} u_{\xi \xi i} u_{\xi \xi i}+2 \zeta^{2} u_{\xi \xi}\left(f_{\xi \xi}+\tilde{D}^{2} u-\beta^{\prime}(u-v)\left(u_{\xi \xi}-v_{\xi \xi}\right)\right) \\
& +C u_{\xi \xi}^{2}-2 \lambda \gamma u_{k i} u_{k i}+2 \lambda u_{k}\left(f_{k}+\tilde{D}^{2} u-\beta^{\prime}(u-v)\left(u_{k}-v_{k}\right)\right) .
\end{aligned}
$$

Choosing $\lambda$ sufficiently large and using also Lemma 3.3, we are led to the inequality

$$
L w \leqslant C-J \text { at } x=x^{0},
$$

where

$$
J=2 \beta^{\prime}(u-v)\left[\zeta^{2} u_{\xi \xi}\left(u_{\xi \xi}-v_{\xi \xi}\right)+\lambda u_{k}\left(u_{k}-v_{k}\right)\right] .
$$

Noting that $u_{\xi \xi} v_{\xi \xi} \leqslant u_{\xi \xi} v_{\xi \xi}^{+}$at $x^{0}$, we find that

$$
J \geqslant \beta^{\prime}(u-v)\left[\zeta^{2} u_{\xi \xi}^{2}-\zeta^{2}\left(v_{\xi \xi}^{+}\right)^{2}+\lambda u_{k} u_{k}-\lambda v_{k} v_{k}\right] \geqslant 0
$$

where the last inequality is a consequence of (4.2).

Since $w$ attains its maximum at $x^{0}$,

$$
L w\left(x^{0}\right)>c\left(x^{0}\right) w\left(x^{0}\right) \geq c_{0} w\left(x^{0}\right) .
$$

But since $L w<C-J \leq C$ at $x^{0}$, we deduce that $w\left(x^{0}\right)<C$. This completes 
the proof that $\|w\|_{L^{\infty}(\Omega)} \leqslant C$. It follows that

$$
\frac{\partial^{2}}{\partial \xi^{2}} u^{k} \leqslant C, \quad 1 \leqslant k \leqslant m, x \in \Omega_{0},
$$

for any direction $\xi$.

For a particular $L^{k}$, choose the coordinate system so that $a_{i j}^{k}=\delta_{i j}$. Then

$$
\begin{aligned}
-\Delta u^{k} & =L^{k} u_{k}+\Sigma b_{i}^{k} \frac{\partial u^{k}}{\partial x_{i}}-c^{k} u^{k} \\
& <f^{k}+\Sigma b_{i}^{k} \frac{\partial u^{k}}{\partial x_{i}}-c^{k} u^{k}<C .
\end{aligned}
$$

By (4.7) we also have

$$
\frac{\partial^{2} u^{k}}{\partial x_{i}^{2}} \leq C \text { for each } i, x \in \Omega_{0} .
$$

It follows that

$$
\frac{\partial^{2} u^{k}}{\partial x_{i}^{2}} \geqslant-(n-1) C, \quad x \in \Omega .
$$

Since $x_{i}$ can be any direction, the second mixed derivatives are also bounded. The proof of the lemma is complete.

5. Passage to the limit as $\varepsilon \rightarrow 0$. In this section we study the "truncated" Bellman equation

$$
\max _{1<k<m}\left(L^{k} u(x)-f^{k}(x)\right)=0 \text { a.e. in } \Omega,
$$

with the boundary condition

$$
u=0 \text { on } \partial \Omega \text {. }
$$

ThEOREM 5.1. Let (1.1)-(1.5) and (1.8) hold. Then there exists a unique solution $u$ of (5.1), (5.2) such that

$$
u \in W^{1, \infty}(\Omega) \cap W_{l o c}^{2, \infty}(\Omega) .
$$

Proof. Denote the solution of (2.3), (2.4) by $u_{\varepsilon}=\left(u_{\varepsilon}^{1}, u_{\varepsilon}^{2}, \ldots, u_{\varepsilon}^{m}\right)$.

From Lemmas 3.3, 4.1 we have the uniform estimates

$$
\begin{array}{ll}
\left|D u_{e}^{k}(x)\right| \leqslant C & (x \in \Omega), \\
\left|D^{2} u_{\varepsilon}^{k}(x)\right| \leqslant C & \left(x \in \Omega_{0}\right)
\end{array}
$$

for any compact domain $\Omega_{0}$ in $\Omega$. Take a sequence of $\varepsilon$ 's (which we denote for simplicity again by $\varepsilon$ ) such that

$$
\begin{gathered}
u_{e}^{k} \rightarrow v^{k}(x) \quad \text { uniformly in } \bar{\Omega}, \\
u_{e}^{k} \rightarrow v^{k}(x) \quad \text { weakly in } W^{2 p}\left(\Omega_{0}\right)
\end{gathered}
$$

for any $p<\infty$ and for any compact subdomain $\Omega_{0} \subset \Omega$. 
From the relation

$$
\beta_{e}\left(u_{e}^{k}-u_{e}^{k+1}\right)=f^{k}-L^{k} u_{e}^{k}
$$

and (5.4), (5.5) it follows that

$$
\beta_{e}\left(u_{\varepsilon}^{k}-u_{\varepsilon}^{k+1}\right)<C \text { in } \Omega_{0} .
$$

Noting that $\beta_{\varepsilon}(t) \rightarrow \infty$ if $t>0, \varepsilon \rightarrow 0$, we deduce that

$$
u_{e}^{k}(x)-u_{\varepsilon}^{k+1}(x) \rightarrow 0 \text { if } \varepsilon \rightarrow 0 ; x \in \Omega .
$$

It follows that the $v^{k}$ defined in (5.6) satisfy $v^{1}=v^{2}=\cdots=v^{m} \equiv u$. We shall prove that

$$
\max _{1<k<m}\left(L^{k} u(x)-f^{k}(x)\right)=0 \text { a.e. in } \Omega \text {. }
$$

We begin by noticing that $L^{k} u_{\varepsilon}^{k}<f^{k}$ and that the weak convergence (in (5.7)) preserves this inequality. Hence

$$
L^{k} u(x)-f^{k}(x)<0 \text { a.e. in } \Omega,
$$

so that also

$$
\max _{1<k<m}\left(L^{k} u(x)-f^{k}(x)\right)<0 \text { a.e. in } \Omega .
$$

To prove the reverse inequality we employ an argument of Evans [9] which generalizes the Minty lemma to the space $C(\bar{\Omega})$. Using the notation of $\$ 2$ we have, by the accretiveness of $L+B_{e}$,

$$
\begin{aligned}
0 & <\left[u_{\varepsilon}-\tilde{\varphi},\left(L+B_{e}\right) u_{e}-\left(L+B_{\varepsilon}\right) \tilde{\varphi}\right]_{+}=\left[u_{\varepsilon}-\tilde{\varphi}, f-\left(L+B_{\varepsilon}\right) \tilde{\varphi}\right]_{+} \\
& =\left[u_{e}-\tilde{\varphi}, f-L \tilde{\varphi}\right]_{+},
\end{aligned}
$$

the last relation obtained by choosing $\tilde{\varphi}$ with components $\varphi^{i}$ such that $\varphi^{1}=\varphi^{2}=\cdots=\varphi^{m} \equiv \varphi$.

We take $\varphi$ in $C_{0}^{\infty}(\Omega)$. Since $u_{\varepsilon}^{k} \rightarrow u$ uniformly in $\Omega$, taking $\varepsilon \rightarrow 0$ in the last expression in (5.10) gives, according to (2.5) and Lemma 2.1,

$$
0<[\tilde{u}-\tilde{\varphi}, f-L \tilde{\varphi}]_{+}=\max _{1<k<m}\left[u-\varphi, f^{k}-L^{k} \varphi\right]_{+}
$$

where $\tilde{u}$ is the vector with $m$ equal components $u$.

By Lemma 2.2 of [9], for a.e. $x^{0} \in \Omega$ there exists a sequence of functions $\varphi_{j} \in C_{0}^{\infty}(\Omega)$ such that

$$
\begin{aligned}
& D^{\alpha} \varphi_{j}\left(x^{0}\right) \rightarrow D^{\alpha} u\left(x^{0}\right) \quad(0<|\alpha| \leqslant 2), \\
& \varphi_{j}\left(x^{0}\right)-u\left(x^{0}\right)=\left\|\varphi_{j}-u\right\|_{C(\Omega)}>\varphi_{j}(x)-u(x) \\
& \forall x \in \Omega, \quad x \neq x^{0} .
\end{aligned}
$$

Taking $\varphi=\varphi_{j}$ in (5.11) and using (2.7), (5.13), we get

$$
0<\max _{1<k<m}\left(L^{k} \varphi_{j}\left(x^{0}\right)-f^{k}\left(x^{0}\right)\right) .
$$


Taking $j \rightarrow \infty$ and using (5.12), we find that

$$
\max _{1<k<m}\left(L^{k} u\left(x^{0}\right)-f^{k}\left(x^{0}\right)\right)>0 \text { a.e. }
$$

This completes the proof of (5.8).

The proof of uniqueness will be given in $\$ 7$.

REMARK. The applicability of the accretive operator methods to the Bellman equation was first noted by Pliska [15].

6. Existence for the Bellman equation. We shall now consider the "full" Bellman equation

$$
\sup _{1<k<\infty}\left(L^{k} u(x)-f^{k}(x)\right)=0 \text { a.e. in } \Omega
$$

with the boundary condition

$$
u=0 \text { on } \partial \Omega \text {. }
$$

THEOREM 6.1. Let (1.1)-(1.5) ana (1.8) hold. Then there exists a unique solution $u$ of (6.1), (6.2) such that

$$
u \in W^{1, \infty}(\Omega) \cap W_{100}^{2, \infty}(\Omega) .
$$

Proof. Denote the solution of (5.1), (5.2) by $u_{m}$. Then

$$
\left|D u_{m}(x)\right|<C \quad(x \in \Omega)
$$

and, for any compact subdomain $\Omega_{0} \subset \Omega$,

$$
\left|D^{2} u_{m}(x)\right|<C \quad\left(x \in \Omega_{0}\right)
$$

here $C$ is a generic constant independent of $m$.

Let

$$
\begin{aligned}
& A_{m} v(x) \equiv \max _{1<k<m}\left(L^{k} v(x)-f^{k}(x)\right), \\
& A v(x) \equiv \sup _{1<k<\infty}\left(L^{k} v(x)-f^{k}(x)\right) .
\end{aligned}
$$

If $\varphi \in C_{0}^{\infty}(\Omega)$ then the $L^{k} \varphi$ are uniformly bounded and equicontinuous, so that as $m \rightarrow \infty$,

$$
A_{m} \varphi(x) \rightarrow A \varphi(x) \text { uniformly in } \bar{\Omega} \text {. }
$$

Consider $A_{m}$ as an operator in $X=L^{\infty}(\Omega)$ with the domain

$$
D\left(A_{m}\right)=\left\{v \in W_{0}^{1, \infty}(\Omega) \cap W_{\mathrm{loc}}^{2, \infty}(\Omega) ; A_{m} v(x) \in L^{\infty}(\Omega)\right\}
$$

LEMMA 6.2. $A_{m}$ is accretive.

Proof. We recall (see Sato $\left[16\right.$, p. 433]) that the bracket $[,]_{+}$in $X=L^{\infty}(\Omega)$ can be computed by

$$
[f, g]_{+}=\lim _{\varepsilon \downarrow 0} \underset{\Omega(f, \varepsilon)}{\text { ess sup }} g(x) \cdot \operatorname{sgn} f(x) \quad(f \neq 0)
$$


where

$$
\Omega(f, \varepsilon) \equiv\left\{x \in \Omega ;|f(x)|>\|f\|_{L^{\infty}(\Omega)}-\varepsilon\right\} .
$$

Now, if $v, \bar{v} \in D\left(A_{m}\right)$ then $v-\bar{v}$ is continuous in $\bar{\Omega}$; and without loss of generality we may assume that $v-\bar{v}$ takes a positive maximum $\|v-\bar{v}\|_{L^{\infty}(\Omega)}$ in $\bar{\Omega}$. We have proven that $\left[v-\bar{v}, A_{m} v-A_{m} \bar{v}\right]_{+}>0$, or, in view of (6.7), that

$$
\lim _{\varepsilon \downarrow 0} \underset{G_{e}}{\operatorname{ess} \sup }\left(A_{m} v(x)-A_{m} \bar{v}(x)\right) \geqslant 0
$$

where

$$
G_{\varepsilon}=\left\{x ; v(x)-\bar{v}(x) \geqslant \max _{\Omega}|v-\bar{v}|-\varepsilon\right\} .
$$

The maximum principle of Bony [4] (see (2.12)) implies that (6.8) holds if $A_{m}$ is replaced by each $L^{k}$; hence obviously (6.8) holds.

Using Lemma 6.2 we can now pass to the limit with $m \rightarrow \infty$ by an argument similar to that used in Theorem 5.1. Indeed, for any $\varphi \in C_{0}^{\infty}(\Omega)$,

$$
0<\left[u_{m}-\varphi, A_{m} u_{m}-A_{m} \varphi\right]_{+}=\left[u_{m}-\varphi,-A_{m} \varphi\right]_{+} \cdot
$$

In view of (6.4), (6.5), there exists a subsequence of $u_{m}$, which we again denote by $u_{m}$, such that $u_{m} \rightarrow u$ uniformly in $\bar{\Omega}, D u_{m} \rightarrow D u$ in the weak star topology of $L^{\infty}(\Omega), D^{2} u_{m} \rightarrow D^{2} u$ in the weak star topology of $L^{\infty}\left(\Omega_{0}\right)$ (for any $\Omega_{0}$, $\left.\bar{\Omega}_{0} \subset \Omega\right)$.

Taking $m \rightarrow \infty$ in (6.9) we obtain, using (6.6) and (2.5), $[u-\varphi,-A \varphi]_{+}>$ 0. Choosing $\varphi=\varphi_{j}$ as in the proof of Theorem 5.1, we get $A \varphi_{j}\left(x^{9}\right)>0$.

Now, since

$$
D^{\alpha} \varphi_{j}\left(x^{0}\right)-D^{\alpha} u\left(x^{0}\right) \rightarrow 0 \quad(0<|\alpha|<2)
$$

and the coefficients of $L^{k}$ are uniformly bounded, we conclude that

$$
A \varphi_{j}\left(x^{0}\right)-A u\left(x^{0}\right) \rightarrow 0 .
$$

It follows that $A u\left(x^{0}\right) \geq 0$.

Conversely, since $L^{k} u_{m}-f^{k}<0$ a.e. in $\Omega, k<m$, we obtain

$$
L^{k} u-f^{k} \leqslant 0 \text { a.e. in } \Omega \text {. }
$$

Hence $A u<0$ a.e. in $\Omega$. We have thus proved that $u$ is a solution of (6.1), (6.2) satisfying (6.3).

The uniqueness of the solution will be proved in $\$ 7$.

REMARK 1. The assumption $c_{0}>0$ made in $\$ \$ 2-6$ may be replaced by $c_{0}>0$. Indeed, if $c_{0}=0$, we observe that the maximum principle still applies to $L-\delta I$ provided $\delta$ is positive and sufficiently small. Hence Theorem 2.3 remains valid (with $L-\delta I+B_{\varepsilon} m$-accretive). Next, (3.1) holds with $1 / c_{0}$ replaced by some positive constant $C$. The remaining arguments now proceed as before. Thus, Theorem 6.1 remains true also when $c_{0}=0$. 
REMARK 2. As a special case of Theorem 6.1 one can immediately establish the existence and uniqueness of a solution for the problem

$$
\begin{gathered}
\lambda u-F\left(u_{x_{1} x_{j}}\right)=f(x) \text { in } \Omega, \\
u=0 \text { on } \partial \Omega
\end{gathered}
$$

provided $\lambda \geqslant 0$ and $F\left(x_{i j}\right)$ satisfies the following conditions:

(a) $F: R^{n^{2}} \rightarrow R$ is convex and $C^{2}$,

(b) $\Sigma F_{x_{i j}} \xi_{i} \xi_{j} \geqslant \gamma|\xi|^{2}, \gamma \geqslant 0$ (ellipticity),

(c) $\left|F(x)-\Sigma F_{x_{i j}}(x) x_{i j}\right|<C \forall x=\left(x_{i j}\right)$.

Indeed, writing

$$
F(x)=\max _{\xi}\left[F(\xi)+F_{x_{i j}}(\xi) \cdot\left(x_{i j}-\xi_{i j}\right)\right], \quad \xi=\left(\xi_{i j}\right),
$$

we may rewrite (6.10) as a Bellman equation with

$$
\begin{gathered}
L^{\xi} \equiv \lambda u-\Sigma F_{x_{i j}}(\xi) \frac{\partial^{2} u}{\partial x_{i} \partial x_{j}}, \\
f^{\xi}(x)=f(x)+F(\xi)-\Sigma F_{x_{i j}}(\xi) \xi_{i j} ;
\end{gathered}
$$

it suffices to let $\xi$ range over a dense countable set in $R^{n^{2}}$.

7. Probabilistic interpretation and methods. Let $k_{1}, \ldots, k_{m}$ be positive numbers. Consider the system of $m$ variational inequalities

$$
\begin{gathered}
L^{i} u_{i}<f^{i} \text { a.e. in } \Omega, \\
u_{i}<k_{i}+u_{i+1} \text { in } \Omega, \\
\left(L^{i} u_{i}-f^{i}\right)\left(u_{i}-k_{i}-u_{i+1}\right)=0 \quad \text { a.e. in } \Omega, \quad 1<i<m .
\end{gathered}
$$

This system is the (formal) limit of the penalized problem

$$
\begin{gathered}
L^{i} u_{i}+\beta_{\varepsilon}\left(u_{i}-k_{i}-u_{i+1}\right)=f^{i} \quad \text { in } \Omega, \\
u_{i}=0 \text { on } \partial \Omega, \quad 1<i<m,
\end{gathered}
$$

where $\beta_{e}(t)$ is the function constructed in $\$ 2$.

We shall need the conditions

$$
\begin{aligned}
& \left|a_{i j}^{k}\right|,\left|b_{i}^{k}\right|,\left|c^{k}\right|,\left|f^{k}\right|<C, \\
& \left|D a_{i j}^{k}\right|,\left|D b_{i}^{k}\right|,\left|D c^{k}\right|<C .
\end{aligned}
$$

Under these assumptions and (1.1), (1.2), (1.4) and the additional condition that $\left|D f^{k}\right|<C$ one can establish the estimates of $\$ 3$ for the solution $u_{i}=u_{i, e}$ of (7.2) (cf. the proof of Theorem 7.2 below). These estimates will provide a uniform modulus of continuity for solutions of (7.2) and these, in turn, imply as we shall see below uniform $W^{2 p}(\Omega)$ estimates (without using condition (1.8)).

Let us now interpret (7.1) probabilistically (cf. Bensoussan and Lions [2] for the case $m=2$ ). This approach gives heuristic insight into the stochastic 
control situation modeled by (7.1) (and (1.6), (1.7)). As a bonus we shall derive the $W^{2, p}(\Omega)$ estimate for solutions of (7.2) under somewhat weaker assumptions than those for the plan outlined above.

Consider the stochastic differential system

$$
d \xi^{i}(t)=\sigma^{i}(\xi(t)) d w(t)+b^{i}(\xi(t)) d t
$$

where $\sigma^{i}(x)$ is a symmetric matrix such that $\frac{1}{2}\left(\sigma^{i}(x)\right)^{2}$ is the matrix $\left(a_{j k}(x)\right)$ and $b^{i}(x)$ is the vector with components $b_{j}^{i}(x)$. Here $w(t)$ is an $n$-dimensional Brownian motion. Denote by $\mathscr{F}_{t}$ the $\sigma$-field $\sigma(w(s), 0<s<t)$.

For any sequence $\theta=\left(\theta_{1}, \theta_{2}, \ldots\right)$ of $\mathscr{F}_{t}$ stopping times $\theta_{1}<\theta_{2}<\cdots<\theta_{l}$ $<\cdots\left(\theta_{l} \uparrow \infty\right.$ if $\left.l \uparrow \infty\right)$ we define the following process $\xi(t)$ :

$$
\xi(t)=\xi^{j}(t) \text { if } \theta_{l m+j-1}<t<\theta_{l m+j} \quad\left(\theta_{0}=0\right)
$$

where $l$ is a nonnegative integer, and $\xi(t)$ is continuous at $t=\theta_{i}$ for all $i$. Thus, $\xi(t)$ switches cyclically from the stochastic system with generator $L^{i}$ to the stochastic system with generator $L^{i+1}\left(L^{m+1} \equiv L^{1}\right)$, and it starts with $\xi(t)=\xi^{l}(t)$ if $0 \leqslant t \leqslant \theta_{1}, \xi(0)=x$. We shall assume for simplicity that $c^{i}(x) \equiv$ const $=\alpha>0$.

Introduce the cost function

$$
J_{x}^{1}(\theta) \equiv E_{x}\left[\int_{0}^{T} e^{-\alpha t} f(\xi(t)) d t+\sum_{l=0}^{\infty} \sum_{j=1}^{m} k_{j} e^{-\alpha \theta_{l m+j}} I_{\theta_{l m+j}}<T\right]
$$

where $T$ is the exit time from $\Omega$ and

$$
f(\xi(t)) \equiv f^{i}\left(\xi^{i}(t)\right) \quad \text { if } \xi(t)=\xi^{i}(t) .
$$

This cost functional represents a running cost (per unit time) of $f^{i}(x)$ when the system is in state $i$, and a switching cost $k_{i}$ for any transition from state $i$ to state $i+1$, the entire cost discounted with a factor $\alpha$.

Set $u_{1}(x) \equiv \inf _{\theta} J_{x}^{1}(\theta)$. More generally we can define $\xi(t)$ which starts with $\xi(t)=\xi^{i}(t)$ for $0 \leqslant t \leqslant \theta_{1}$, and then proceed cyclically as before to change from any state $j$ to state $j+1$. We denote the corresponding cost function by $J_{x}^{i}(\theta)$, and set

$$
u_{i}(x)=\inf _{\theta} J_{x}^{i}(\theta)
$$

THEOREM 7.1. Assume that (1.1), (1.2), (1.4), (1.5), and (7.3) hold. Then $\left(u_{1}, \ldots, u_{m}\right)$ forms a solution of $(7.1)$, belonging to $C(\bar{\Omega}) \cap W^{2 p}(\Omega)$ for any $p<\infty$. Conversely, every solution of (7.1) which belongs to $C(\bar{\Omega}) \cap W_{\mathrm{loc}}^{2 p}(\Omega)$ for some $p>n / 2$ is given by (7.5) (and is therefore unique).

Proof. The second part of the theorem follows by a standard application of Ito's formula to functions in $C(\bar{\Omega}) \cap W_{\text {loc }}^{2 p}(\Omega)$ (cf. [3] [11]). Thus it remains to prove the existence of a solution of (7.1) which belongs to $W^{2 p}(\Omega)$ for all $p<\infty$. 
We truncate the cost function $J_{x}^{i}(\theta)$ by restricting the number of $\theta_{i}$ 's to be at most $N$. Denote the corresponding cost function by $J_{x}^{i, N}(\theta)$ and set $u_{i}^{N}(x)=\inf _{\theta} J_{x}^{i, N}(\theta)$. Thus, if $N=1$ then $u_{i}^{1}$ is simply a solution of the variational inequality

$$
L^{i} u_{i}^{1}<f^{i}, \quad u_{i}^{1}<k_{i}, \quad\left(L^{i} u_{i}^{1}-f^{i}\right)\left(u_{i}^{1}-k_{i}\right)=0,
$$

that is, when $N=1$ the obstacle for $u_{i}^{N}$ is $k_{i}$.

Similarly, for any $N$, the obstacle for $u_{i}^{N}$ is $k_{i}+u_{i+1}^{N-1}$. This in fact can be proved in a standard way by the principle of dynamic programming, using the strong Markov property.

It is well known (see, for instance, [3]) that if the obstacle is continuous then the solution of the variational inequality is also continuous. Hence by induction it follows that the $u_{i}^{N}$ are all continuous functions in $\bar{\Omega}$.

We claim that

$$
\left|u_{i}^{N}(x)-u_{i}(x)\right|<C / N \quad(x \in \bar{\Omega})
$$

where $u_{i}(x)$ is defined by (7.5).

Notice first that

$$
u_{i}(x) \leqslant u_{i}^{N}(x)
$$

since any $\operatorname{cost} J_{x}^{i, N}(\theta)$ is also a cost $J_{x}^{i}(\theta)$ (with $\theta_{j}=\infty$ if $j>N$ ).

Next, in estimating $u_{i}(x)$ from below we may restrict ourselves only to "good" choices of stopping times $\theta_{i}$. More specifically, since we may always choose not to switch, it is clear that we may restrict the $\theta_{i}$ to be such that

$$
E_{x}\left[\sum_{l m+j>i} k_{j} e^{-\alpha \theta_{l m+j}} I_{\theta_{l m+j}}<T\right]<E_{x}\left[\int_{\theta_{i}}^{T} e^{-\alpha t} f(\xi(t)) d t\right] .
$$

Recalling that $f$ is bounded, we conclude that in computing $u_{i}(x)$ it suffices to take the infimum on $\theta$ with $\theta_{k}$ satisfying:

$$
\sum_{i>j} E_{x}\left[e^{-\alpha \theta_{i}} I_{\theta_{i}<T}\right]<C E_{x}\left[e^{-\alpha \theta_{j}} I_{\theta_{j}<T}\right]
$$

for some $C$.

We may also restrict the $\theta_{k}$ to be such that $J_{x}^{i}(\theta)<C$ for some $C$ sufficiently large. In view of (7.8) and the boundedness of $f$, this implies that

$$
\sum_{i} E_{x}\left[e^{-\alpha \theta_{i}} I_{\theta_{i}<T}\right]<C .
$$

Since the $i$ th term is nonnegative and decreasing, it follows that

$$
j E_{x}\left[e^{-\alpha \theta_{j}} I_{\theta_{j}<T}\right]<C .
$$

Using (7.8), (7.9) it follows that $\left|J_{x}^{i}(\theta)-J_{x}^{i, N}(\tilde{\theta})\right|<C / N$ for any $\theta=$ $\left(\theta_{1}, \theta_{2}, \ldots\right)$, where $\tilde{\theta}$ is obtained from $\theta$ by dropping all the $\theta_{j}$ with $j>N$. It follows that $u_{i}^{N}(x)-u_{i}(x)<C / N$, and recalling (7.7), the assertion (7.6) follows. 
Since $u_{i}^{N}(x)$ are continuous in $\bar{\Omega},(7.6)$ implies equicontinuity of the $u_{i}^{N}(x)$ and the continuity of $u_{i}(x)$ in $\bar{\Omega}$.

At each point $x^{0} \in \Omega$ we cannot have $u_{i}\left(x^{0}\right)=k_{i}+u_{i+1}\left(x^{0}\right)$ for all $1<i$ $\leqslant m$. Hence there exist $\varepsilon_{0}>0, \delta>0$ and some $i$ such that

$$
u_{i}(x)-k_{i}-u_{i+1}(x)<-\varepsilon_{0} \text { if }\left|x-x^{0}\right|<\delta .
$$

It follows that $u_{i}^{N}(x)<k_{i}+u_{i+1}^{N-1}-\varepsilon_{0} / 2$ if $\left|x-x^{0}\right|<\delta$ if $N$ is sufficiently large, say $N>N_{0}$. Hence

$$
L^{i} u_{i}^{N}=f^{i} \quad \text { if }\left|x-x^{0}\right|<\delta, \quad N>N_{0} .
$$

Since $u_{i}^{N}$ is uniformly bounded, the standard elliptic estimates give $\left\|u_{i}^{N}\right\|_{W^{2 \rho}\left(G_{\delta}\right)}<C$ for any $p<\infty, N \geqslant N_{0}$, where $G_{\delta}=\{x ; x \in \Omega,|x-x|$ $<\delta / 2\}$ and $C$ is a constant independent of $N$.

Considering $k_{i-1}+u_{i}^{N}$ as an obstacle in the variational inequality for $u_{i-1}^{N+1}$, we then deduce that $\left\|u_{i-1}^{N+1}\right\|_{W^{2 \rho}\left(G_{6}\right)}<C$ with a smaller $\delta$. Proceeding in this manner step by step $m-1$ times, we find that $\left\|u_{i-j}^{N+j}\right\|_{W^{20}\left(G_{8}\right)}<C$ $\left(1<j<m-1, N \geqslant N_{0}\right)$. Recalling (7.6) we deduce that $\left\|u_{j}\right\|_{W^{2}{ }_{\left(G_{8}\right)}}<C$. Hence $u_{j} \in W^{2, p}(\Omega)$.

Finally, taking $N \rightarrow \infty$ in the variational inequalities for $u_{j}^{N}(1<j<m)$ we find that $\left(u_{1}, \ldots, u_{m}\right)$ is a solution of the system (7.1).

REMARK. Using the $C^{1,1}$ regularity of solutions of variational inequalities [6], the above proof establishes that $u_{i} \in C^{1,1}(\Omega)$ provided the $f^{i}$ are Hölder continuous and $a_{i j}^{k}, b_{i}^{k}$ are in $C^{2}(\bar{\Omega})$.

The probabilistic idea underlying the penalized problem (2.3), (2.4) is that $\beta_{e}\left(u^{k}-u^{k+1}\right)$ represents (in some heuristic sense) a penalty for switching from the stochastic system for $\xi^{k}$ to the stochastic system for $\xi^{k+1}$. In the limit case of the Bellman equation, there is no cost for switching. In fact, Krylov [12] writes the solution $u(x)$ of the Bellman equation (in $R^{n}$ ) as the infimum of the cost functions

$$
E_{x}\left[\int_{0}^{\infty} e^{-\alpha t} \sum_{i=1}^{\infty}\left(f^{o(t)}\left(\xi^{o(t)}(t)\right) I_{v(t)=i}\right) d t\right]
$$

where $v(t)$ is any nonanticipative function with range $1,2, \ldots$ If we restrict $v(t)$ to have the range $1,2, \ldots, m$ and to have only a countable number of discontinuities at times $\theta_{1}<\theta_{2}<\ldots, \theta_{l} \uparrow \infty$, and if further $v\left(\theta_{i}+0\right)=v\left(\theta_{i}\right.$ $-0)+1$ (if $v\left(\theta_{i}-0\right)<m$; $v\left(\theta_{i}+0\right)=1$ if $v\left(\theta_{i}-0\right)=m$ ), then this cost coincides with the cost $J_{x}^{1}(\theta)$ introduced above when $T=\infty$ and with no penalty for switching (i.e., $k_{j}=0$ for all $j$ ).

It thus seems natural to try to obtain the solution $u$ of the Bellman equation (5.1), (5.2) as the limit of the solution of (7.1) as $k=\left(k_{1}, \ldots, k_{m}\right) \rightarrow$ 
0. In fact we have:

THEOREM 7.2. Let (1.1)-(1.5) and (1.8) hold. Then there exists a unique solution $u^{k}=\left(u_{1}^{k}, \ldots, u_{m}^{k}\right)$ of (7.1) with $u_{i}^{k}$ in $W^{1, \infty}(\Omega) \cap W_{\operatorname{loc}}^{2, \infty}(\Omega)$, and

$$
u_{i}^{k}(x) \rightarrow u(x) \text { uniformly in } \bar{\Omega} \text { as } k \rightarrow 0 \text {, }
$$

where $u$ is the solution of (5.1), (5.2).

The proof is similar to the proof of Theorem 5.1. We first consider the system (7.2) and prove that it has a unique solution $\left(u_{1}^{k, \ell}, \ldots, \bar{u}_{m}^{k, e}\right)$. Next we derive estimates on $u_{i}^{k, \varepsilon}$ analogous to those derived in $\$ \S 3$ and 4. In deriving the estimate on $D u_{i}^{k, e}$ we get an inequality analogous to (3.15), with $\beta^{\prime}=\beta^{\prime}(u$ $\left.-v-k_{1}\right)$ and with an extra term $\hat{J}=2 \lambda u k_{1} \beta^{\prime}\left(u-v-k_{1}\right)$ on the righthand side. Without loss of generality we may assume that $u>0$ (otherwise we derive the gradient estimates for $u_{i}^{k, \ell}+C$ instead of $u_{i}^{k, e}$; consequently $\hat{J}>0$, and we then continue as in $\$ 3$. We can now take $\varepsilon \rightarrow 0$ to obtain the solution $\left(u_{1}^{k}, \ldots, u_{m}^{k}\right)$ of (7.1). Finally, passing to the limit with $k \rightarrow 0$ and arguing as in $\S 5$, we find that (7.11) is valid where $u$ solves (5.1), (5.2).

We can actually prove in a similar way that $u_{i}^{k, \ell} \rightarrow u$ if $k \rightarrow 0, \varepsilon \rightarrow 0$. The proof of Theorem 5.1 is in fact the case where $k=0, \varepsilon \rightarrow 0$.

Uniqueness for the Bellman equation. The proof of the uniqueness asserted in Theorem 6.1 is similar to the proof of uniqueness for $R^{n}$ given by Krylov [13] (see also [3, Chapter 4, §5]. One constructs a nearly optimal "feedback control" on a subset of $\Omega$ whose complement in $\Omega$ has a small measure. The crucial step is in being able to apply Ito's formula for a function in $W_{100}^{2 p}(\Omega) \cap$ $C(\bar{\Omega})(p>n)$ for a process $d \xi(t)=\sigma(t) d w(t)+b(t) d t$ where $\sigma(t), b(t)$ are bounded and nonanticipative. This, in turn, is established by using Krylov's inequality [12]

$$
\left|E_{x}\left[\int_{0}^{T_{0}} \varphi(\xi(t)) d t\right]\right|<C\|\varphi\|_{L^{P}\left(\Omega_{0}\right)}
$$

where $\Omega_{0}$ is any domain with $\bar{\Omega}_{0} \subset \Omega$ and $T_{0}$ is the exit time from $\Omega_{0} ; C$ is a constant depending only on a bound on $\sigma, b, \sigma^{-1}$ and the diameter of $\Omega$.

One can represent the solution of (5.1), (5.2) in the form (we take for simplicity $\left.c^{i}(x) \equiv \alpha\right)$

$$
u(x)=\inf _{\theta} E_{x}\left[\int_{0}^{T} e^{-\alpha t} f(\xi(t)) d t\right]
$$

where $f(\xi(t))$ is defined by (7.4), $\theta$ is any sequence of stopping times $\theta_{i}$ increasing to $\infty$ and $\xi(t)$ switches from each $\xi^{j}$ to $\xi^{k}$ according to the rule: $\xi^{1}$, $\xi^{2}, \xi^{1}, \xi^{2}, \xi^{3}, \xi^{1}, \xi^{2}, \xi^{3}, \xi^{4}, \xi^{1}, \ldots$ This fact follows from the probabilistic interpretation of the solution of the "truncated" Bellman equation.

In the uniqueness proof for the Bellman equation one can choose a nearly optimal "feedback control" $v(t)$ on a subset of $\Omega$ whose complement has a 
small measure as follows: $v(t)$ assigns the state $\xi^{i}(t)$ where $\xi(t)$ is in closed set $A_{i} \subset \Omega$, and the $A_{i}$ are disjoint sets. Using such controls, we can easily give another proof of the assertion (7.12).

REMARK. Uniqueness for a solution of the truncated Bellman equation (5.1) is also immediate from the maximum principle of Bony [4].

We conclude this section with a generalization of Theorem 7.1 to the system of variational inequalities

$$
\begin{gathered}
L^{i} u_{i}+\sum_{j=1}^{m} \beta\left(u_{i}-u_{j}-k_{i j}\right) \ni f^{i} \quad \text { a.e. in } \Omega \\
u_{i}=0 \quad \text { on } \partial \Omega \quad(1<i \leqslant m)
\end{gathered}
$$

where $k_{i j}>0$ if $i \neq j, k_{i i}=0$.

We can again give a probabilistic interpretation of the solution (the switchings cost $k_{i j}$ and need not be cyclic) and prove existence and uniqueness as for Theorem 7.1. Another way to prove existence is to derive estimates on the first derivatives of the solution of the penalized system

$$
\begin{gathered}
L^{i} u_{i}+\sum_{j=1}^{m} \beta_{e}\left(u_{i}-u_{j}-k_{i j}\right)=f^{i} \quad \text { in } \Omega, \\
u_{i}=0 \quad \text { on } \partial \Omega \quad(1<i<m)
\end{gathered}
$$

by the method of $\$ 3$ and then proceed as in the proof of Theorem 7.1 to derive $W^{2 p}$ estimates. [The method of $\$ 3$ requires the additional assumption that $\left|D f^{i}\right|<C$.]

In establishing the $W^{2 p}$ estimates let us notice that for any $x^{0} \in \Omega$ we may assume that $u_{1} \leqslant u_{2} \leqslant \cdots \leqslant u_{m}$ at $x^{0}$, and therefore

$$
\beta_{e}\left(u_{i}-u_{j}-k_{i j}\right)=0 \text { if } j>i
$$

in $G_{\delta}=\left\{\left|x-x^{0}\right|<\delta\right\} \cap \Omega$ for some $\delta>0$ and all $\varepsilon$ sufficiently small. Therefore

$$
\begin{gathered}
L^{1} u_{1}=f^{1} \\
L^{i} u_{i}+\sum_{j=1}^{i-1} \beta_{\varepsilon}\left(u_{i}-u_{i}-k_{i j}\right)=f^{i}
\end{gathered}
$$

for $2<i<m$.

Clearly $u_{1} \in W_{\text {loc }}^{2 p}\left(G_{\delta}\right)$. We can now prove by induction that $u_{i} \in W_{\text {loc }}^{2 p}\left(G_{\delta}\right)$. Indeed, to pass from $i-1$ to $i$, we set $\varphi_{j}=u_{j}+k_{i j}(1<j<i-1)$ and multiply (7.16) by $\zeta^{p} \beta_{\varepsilon}^{p-1}\left(u_{i}-\varphi_{k}\right)(1<k<i)$, where $\zeta$ is a cutoff function 
with support in $G_{\delta}$. By integration by parts,

$$
\begin{aligned}
& \int L^{i} u_{i} \zeta^{P} \beta_{e}^{p-1}\left(u_{i}-\varphi_{k}\right) \\
&=\int L^{i}\left(u_{i}-\varphi_{k}\right) \zeta^{p} \beta_{e}^{p-1}\left(u_{i}-\varphi_{k}\right)+\int L^{i} \varphi^{k} \cdot \zeta^{P} \beta_{e}^{p-1}\left(u_{i}-\varphi_{k}\right) \\
&>-C \int \zeta^{p-1}|\nabla \zeta| \beta_{e}^{p-1}\left(u_{i}-\varphi_{k}\right) \\
&-\eta \int \zeta^{p} \beta_{e}^{p}\left(u_{i}-\varphi_{k}\right)-C(\eta) \int \zeta^{p}\left|L^{i}\left(\varphi_{k}\right)\right|^{p} \\
& \geqslant-C(\eta)-2 \eta \int \zeta^{P} \beta_{e}^{P}\left(u_{i}-\varphi_{k}\right) \quad(\forall \eta>0) .
\end{aligned}
$$

Since

$$
\beta_{e}\left(u_{i}-\varphi_{j}\right) \beta_{e}^{p-1}\left(u_{i}-\varphi_{k}\right)>0,
$$

we easily get

$$
\int \zeta^{p} \beta_{e}^{p}\left(u_{i}-\varphi_{k}\right)<C .
$$

It follows that $L^{i} u_{i} \in L_{\text {loc }}^{p}\left(G_{\delta}\right)$, so that $u_{i} \in W_{\text {loc }}^{2 p}\left(G_{\delta}\right)$.

We have proved:

ThEOREM 7.3. Assume that (1.1), (1.2), (1.5) and (7.3) hold. Then there exists a unique solution of (7.13) whose components belong to $W^{2 p}(\Omega)$ for any $p<\infty$.

8. The case of two operators. Consider now the system of two variational inequalities

$$
\begin{gathered}
L^{1} u^{1}+\beta\left(u^{1}-u^{2}-k_{1}\right) \ni f^{1} \text { in } \Omega, \\
L^{2} u^{2}+\beta\left(u^{2}-u^{1}-k_{2}\right) \ni f^{2} \text { in } \Omega, \\
u^{1}=u^{2}=0 \text { on } \partial \Omega
\end{gathered}
$$

where $k_{1}>0, k_{2}>0, \beta(t)=\{0\}$ if $t<0, \beta(0)=[0, \infty]$. Consider also the Bellman equation

$$
\begin{gathered}
\max \left\{L^{1} u(x)-f^{1}(x), L^{2} u(x)-f^{2}(x)\right\}=0 \text { in } \Omega, \\
u=0 \text { on } \partial \Omega .
\end{gathered}
$$

We assume, in addition to (1.1)-(1.3) that

$$
\begin{gathered}
f^{i} \in W^{1, p}(\Omega) \quad \text { for some } p>n, \\
c^{1}(x)>\alpha_{0}, \quad c^{2}(x) \geqslant \alpha_{0},
\end{gathered}
$$

where $\alpha_{0}$ is sufficiently large depending on the coefficients of $L^{i}$ and on $\Omega$. Then [5] there exists a unique solution $u$ of (8.2) in $H^{3}(\Omega) \cap H_{0}^{1}(\Omega)$, and it belongs to $C^{2, \alpha}\left(\Omega_{0}\right)$ for some $\alpha>0$ and for any domain $\Omega_{0}$ with $\bar{\Omega}_{0} \subset \Omega$. Further, by [10], $u \in C(\bar{\Omega})$. 
We denote the solution of $(8.1)$ by $\left(u_{k}^{1}, u_{k}^{2}\right)$ where $k=\left(k_{1}, k_{2}\right)$.

THEOREM 8.1. If (1.1)-(1.3) and (8.3), (8.4) hold, then

$$
u_{k}^{i}(x) \rightarrow u(x) \text { weakly in } H^{2}(\Omega) \text { as } k \rightarrow 0 .
$$

This theorem was stated in $\$ 7$ for a general system (see Theorem 7.2) in case $\alpha_{0}$ is any positive number, but only where the coefficients $a_{i j}^{k}(x)$ are constants.

Proof. We shall use below an inequality of Sobolevsky [18] (for proof see, for instance, [5]):

$$
\int_{\Omega} L^{1} v \cdot L^{2} v d x \geqslant C\|v\|_{H^{2}(\Omega)}^{2} \quad \forall v \in H^{2}(\Omega) \cap H_{0}^{1}(\Omega), \quad C>0 .
$$

Clearly (8.1) implies

$$
k_{2} \leqslant u_{k}^{1}(x)-u_{k}^{2}(x)<k_{1} .
$$

We shall now prove that

$$
\left\|u_{k}^{i}\right\|_{H^{2}(\Omega)} \leqslant C \quad(i=1,2)
$$

where $C$ is a generic positive constant independent of $k$.

Set for simplicity $u_{k}^{1}=u, u_{k}^{2}=v, L^{1}=L, L^{2}=M, f^{1}=f, f^{2}=g$ and

$$
\gamma_{i}=f^{i}-L^{i} u_{k}^{i} \in \beta\left(u^{i}-u^{j}-k_{i}\right) \quad(i, j=1,2 ; i \neq j) .
$$

Then (8.1) may be written in the form $L u+\gamma_{1}=f, M v+\gamma_{2}=g$ a.e. in $\Omega$. It follows that

$$
\begin{gathered}
\int_{\Omega} L u(M u-M v)+\int_{\Omega} \gamma_{1}(M u-M v)=\int_{\Omega} f(M u-M v), \\
\int_{\Omega} M v(L v-L u)+\int_{\Omega} \gamma_{2}(L v-L u)=\int_{\Omega} g(L v-L u) .
\end{gathered}
$$

By the coercivity of $M$ and $L$ it follows that the second terms on the left-hand sides of (8.9), (8.10) are nonnegative (cf. Brezis-Strauss [7, Lemma 2]).

Adding (8.9), (8.10) and using the Sobolevsky inequality we get

$$
C\left(\|u\|_{H^{2}}^{2}+\|v\|_{H^{2}}^{2}\right) \leqslant \int_{\Omega} f(M u-M v)+\int g(L v-L u)+2 \int_{\Omega} M v L u .
$$

Observe next that $\gamma_{1} \gamma_{2}=0$ a.e., and so $(L u-f)(M v-g)=0$ a.e. Hence

$$
\int_{\Omega} M v L u \leqslant \varepsilon\left(\|u\|_{H^{2}}^{2}+\|v\|_{H^{2}}^{2}\right)+\frac{C}{\varepsilon} \int_{\Omega}\left(f^{2}+g^{2}\right) .
$$

Using this in (8.11), the assertion (8.8) follows.

We can now take a subsequence of $\left(u_{k}, v_{k}\right)$, which we again denote by $\left(u_{k}, v_{k}\right)$, such that (recall (8.7))

$$
u_{k}, v_{k} \rightarrow w \quad \text { weakly in } H^{2}(\Omega) .
$$


Since $L u_{k}<f, M v_{k}<g$, we get $L w \leqslant f, M w<g$, so that

$$
\Phi \equiv \max (L w-f, M w-g)<0, \quad w \in H^{2}(\Omega) \cap H_{0}^{1}(\Omega) .
$$

We shall prove that $w=u$, where $u$ is the solution of (8.2).

From the probabilistic interpretation of $u_{k}, v_{k}$ we deduce that

$$
u \leqslant u_{k}, \quad u \leqslant v_{k}
$$

therefore

$$
u<w .
$$

This we can also prove by the maximum principle. Indeed, if (8.14) is not true then without loss of generality we may assume that there exists a point $x^{0} \in \Omega$ such that

$$
\begin{gathered}
\left(u-u_{k}\right)\left(x^{0}\right)=\max _{\Omega}\left(u-u_{k}\right)>0, \\
\max _{\bar{\Omega}}\left(u-u_{k}\right)>\max _{\Omega}\left(u-v_{k}\right) .
\end{gathered}
$$

Then, by Bony [4],

$$
f\left(x^{0}\right) \geqslant L u\left(x^{0}\right)>\text { ess } \lim _{x \rightarrow x^{0}} \sup L u_{k}(x)=f\left(x^{0}\right)-\text { ess } \lim _{x \rightarrow x_{0}} \inf \gamma_{1},
$$

so that ess lim inf $\gamma_{1}>0$. Hence $u_{k}\left(x^{0}\right)>v_{k}\left(x^{9}\right)$. It follows that $\left(u-v_{k}\right)\left(x^{9}\right)$ $>\left(u-u_{k}\right)\left(x^{9}\right)$, which contradicts (8.16), (8.17).

Having proved (8.15), it remains to prove that $w>u$. Notice, by (8.13), that

$$
\max \{L w-(f+\Phi), M w-(g+\Phi)\} \equiv 0
$$

and $f+\Phi \leqslant f, g+\Phi \leqslant g$. Hence it suffices to prove a comparison theorem for the Bellman equation, namely,

$$
\text { if } f<\tilde{f}, g<\tilde{g} \text { then } \tilde{u}<u
$$

where $\tilde{u}$ is the solution corresponding to $\tilde{f}, \tilde{g}$.

For smooth $f_{2} \tilde{f}, g, \tilde{g}$ the proof follows by the probabilistic interpretation of $u$, $\tilde{u}$ or by a simple maximum principle argument using the $C^{2+\alpha}(\Omega) \cap C(\bar{\Omega})$ regularity of $u$ and $\tilde{u}$.

To prove (8.18) for general $f \tilde{f}, g, \tilde{g}$, we approximate these functions by smooth functions and use the following lemma.

LEMMA 8.2. Let $\max \left(L u_{m}-f_{m}, M u_{m}-g_{m}\right)=0$ a.e. in $\Omega, u_{m} \in H^{2}(\Omega) \cap$ $H_{0}^{1}(\Omega)$. If $f_{m} \rightarrow f, g_{m} \rightarrow g$ in $L^{2}(\Omega)$, then $u_{m} \rightarrow u$ in $H^{2}(\Omega)$.

Proof. We have $L u_{m}+\beta\left(M u_{m}-g_{m}\right) \ni f_{m}, L u+\beta(M u-g) \ni f$. Hence

$$
L\left(u_{m}-u\right)+\gamma_{m}-\gamma=f_{m}-f
$$

where $\gamma_{m} \in \beta\left(M u_{m}-g_{m}\right), \gamma \in \beta(M u-g)$. Multiplying (8.19) by $M\left(u_{m}-u\right)$ $+g-g_{m}$ and integrating over $\Omega$ we obtain, after using the Sobolevsky 
inequality,

$$
\begin{aligned}
C\left\|u_{m}-u\right\|_{H^{2}}^{2} \leqslant & \frac{C}{\varepsilon}\left(\left\|f_{m}-f\right\|_{L^{2}}^{2}+\left\|g_{m}-g\right\|_{L^{2}}^{2}\right)+\varepsilon\left\|u_{m}-u\right\|_{H^{2}}^{2} \\
& -\int_{\Omega}\left(\gamma_{m}-\gamma\right)\left(M\left(u_{m}-u\right)+g-g_{m}\right) .
\end{aligned}
$$

Since the last integral is nonnegative (by the monotonicity of $\beta$ ), the assertion of the lemma follows.

\section{REFERENCES}

1. V. Barbu, Nonlinear semigroups and differential equations in Banach spaces, Noordhoff, Leyden, The Netherlands, 1976.

2. A. Bensoussan and J. L. Lions, Contrôle impulsionnel et systèmes d'inéquations quasi variationneles, C. R. Acad. Sci. Paris 278 (1974), 747-751.

3. Applications des inéquations variationelles en contrôle stochastique, Dunod, Paris, 1978.

4. J.-M. Bony, Principle du maximum dans les espaces de Soboleo, C. R. Acad. Sci. Paris 265 (1967), 333-336.

5. H. Brezis and L. C. Evans, A variational inequality approach to the Bellman-Dirichlet equation for two elliptic operators, Arch. Rational Mech. Anal. (to appear).

6. H. Brézis and D. Kinderlehrer, The smoothness of solutions to nonlinear variational inequalities, Indiana Univ. Math. J. 9 (1974), 831-844.

7. H. Brézis and W. Strauss, Semi-linear second order elliptic equations in $L^{1}$, J. Math. Soc. Japan 25 (1973), 565-590.

8. M. Chicco, Principio di massimo per soluzioni di equazioni ellittiche del secondo ordine di tipo Cordes, Ann. Mat. Pura Appl. (4) 100 (1974), 239-258.

9. L. C. Evans, A convergence theorem for solutions of nonlinear second order elliptic equations, Indiana Univ. Math. J. 27 (1978), 875-887.

10. L. C. Evans and P. L. Lions, Dewx résultats de régularité pour le problème de Bellman-Dirichlet, C. R. Acad. Sci. Paris Sér. A-B 286 (1978), 587-589.

11. A. Friedman, Stochastic differential equations and applications. vol. II, Academic Press, New York, 1976.

12. N. V. Krylov, An inequality in the theory of stochastic integrals, Theor. Probability Appl. 16 (1971), 438-448.

13. $\ldots$, Control of a solution of a stochastic integral equation, Theor. Probability Appl. 17 (1972), 114-130.

14. M. Nisio, Remarks on stochastic optimal controls, Japan J. Math. 1 (1975), 159-183.

15. S. Pliska, A semigroup representation of the maximum expected reward vector in continuous parameter Markoo decision theory, SIAM J. Control 13 (1975), 1115-1129.

16. K. Sato, On the generators of non-negative contraction semigroups in Banach spaces, J. Math. Soc. Japan 20 (1968), 423-436.

17. E. Sinestrani, Accretive differential operators, Boll. Un. Mat. Ital. 13 (1976), 19-31.

18. P. Sobolevsky, Equations with operators constituting an acute angle, Dokl. Akad. Nauk SSSR 116 (1957), 754-757. (Russian)

Department of Mathematics, Untversity of Kentucky, LeXongton, Kentucky 40506

Departiment of Mathematics, Northwestern Universtry, Evanston, ILLNOIS 60201 